\title{
Metas de Realização entre Estudantes do Ensino Médio: Evidências de Validade Fatorial e Consistência Interna de uma Medida ${ }^{1}$
}

\author{
Valdiney V. Gouveia ${ }^{2}$ \\ Pollyane K. da Costa Diniz \\ Walberto Silva dos Santos \\ Rildésia S. V. Gouveia \\ Jane Palmeira Nóbrega Cavalcanti \\ Universidade Federal da Paraíba
}

\begin{abstract}
RESUMO - O presente estudo teve como objetivo principal conhecer evidências de validade fatorial e consistência interna do Questionário de Metas de Realização para o contexto brasileiro. Complementarmente, procurou avaliar em que medida as pontuações nesse instrumento se correlacionam com indicadores de desempenho escolar. Participaram da pesquisa 307 estudantes do ensino médio de duas escolas públicas (68\%) e uma particular (32\%) da cidade de João Pessoa. Esses tinham idade média de 17,6 anos $(D P=3,94)$, sendo a maioria do sexo feminino (61,2\%). Os resultados da análise de Componentes Principais indicaram a pertinência de assumir uma estrutura multifatorial, com quatro componentes teorizados de metas: aprendizagemaproximação, aprendizagem-evitação, execução-aproximação e execução-evitação. Esses componentes foram corroborados por meio de análises fatoriais confirmatórias, que testaram diferentes modelos alternativos. As metas de aprendizagem-aproximação caracterizaram a maioria dos participantes e suas pontuações foram positivamente correlacionadas com o desempenho escolar. Esses resultados foram discutidos tomando em conta a literatura acerca das metas de realização.
\end{abstract}

Palavras-chave: metas; realização; motivação; desempenho escolar; questionário.

\section{Achievement Goals among High School Students: Evidences of Factorial Validity and Internal Consistency of a Measure}

\begin{abstract}
The present study aimed at knowing evidences of factorial validity and internal consistency of the Achievement Goals Questionnaire to the Brazilian milieu. Moreover, it attempted to evaluate to what extent the scores on this instrument correlate themselves with indicators of scholar performance. The participants were 307 high school students from two public schools $(68 \%)$ and one private school $(32 \%)$, from João Pessoa city. Their mean age was $17.6(S D=3.94)$, and the most of them were female (61.2\%). Results of the Principal Components analysis suggested a multi-factor structure, with four theorized components of goals: mastery-approach, mastery-avoidance, performance-approach, and performance-avoidance. All components were corroborated by confirmatory factor analyses, which tested different alternative models. The masteryapproach goals described most of the participants, and their scores were positively correlated with scholar performance. These findings were discussed based on the literature about achievement goals.
\end{abstract}

Keywords: goals; achievement; motivation; scholar performance; questionnaire.

No processo de ensino-aprendizagem, o aspecto motivacional parece crucial (Covington, 2000). Embora contribua a motivação do professor para ensinar, talvez mais fundamental seja o empenho dos alunos em aprender e as estratégias adotadas com tal finalidade. Um construto importante nesse contexto diz respeito à orientação à meta, isto é, o motivo básico que leva os indivíduos a demonstrarem competência ou realização. A orientação à meta, ou simplesmente meta de realização, tem despertado o interesse de pesquisadores da Educação e Psicologia (Dowson \& McInerney, 2004;

1 Durante a preparação deste artigo, o primeiro autor contou com bolsa de produtividade, enquanto o segundo e o último contaram com bolsa de iniciação científica $(\mathrm{CNPq})$; o terceiro e o quarto autores contaram com bolsa de doutorado e mestrado (CAPES), respectivamente. Os autores agradecem a essas instituições.

2 Endereço: Universidade Federal da Paraíba, CCHLA - Departamento de Psicologia. João Pessoa, PB. CEP 58051-900 E-mail:vgouveia@cchla.ufpb.brou vvgouveia@pesquisador.cnpq.br.
Dupeyrat \& Mariné, 2005; Jagacinski \& Duda, 2001; Pintrich, Conley \& Kempler, 2003). Compreende a orientação adotada pelo indivíduo diante de uma tarefa que demanda demonstrar competência. As metas de realização são vistas como o propósito ou foco cognitivo-dinâmico de engajamento em uma tarefa, sendo que o tipo de meta adotado fornece o referencial que permite ao indivíduo interpretar e experimentar os contextos de realização (Elliot \& McGregor, 2001; Elliot, McGregor \& Gable, 1999).

As metas de realização são fundamentais para explicar o comportamento dos indivíduos em tarefas de desempenho, como aquelas típicas do contexto escolar. Portanto, conhecer as metas adotadas pelos indivíduos pode ser útil para estimar seu esforço em aprender e mesmo os resultados acadêmicos alcançados (Elliot \& cols., 1999; Puca \& Schmalt, 1999). Nesse sentido, faz-se mister contar com instrumentos psicometricamente adequados, tipo lápis e papel, que sejam breves e de fácil aplicação. 
Procurando na literatura brasileira, especificamente na base de dados Biblioteca Virtual em Saúde - Psicologia (2006), foram identificadas 21 referências com a expressão metas de realização. No entanto, desse total, apenas três referiam-se à temática no âmbito escolar, propondo medidas a respeito (Bueno, Zenorine, Santos, Matumoto \& Buchatsky, 2007; Cardoso \& Bzuneck, 2004; Zenorini, Santos \& Bueno, 2003). Não obstante, essas referências avaliam unicamente dois componentes das metas (aprendizagem e execução) ou, no máximo, três componentes (aprendizagem, execução-aproximação e execução-evitação), não permitindo cobrir avanços teóricos importantes acerca desse atributo psicológico (Elliot \& McGregor, 2001). Essa situação sugeriu, assim, procurar alguma medida que pudesse ser adaptada de outro contexto cultural. Tais aspectos motivaram o presente estudo, cujo objetivo principal foi adaptar o Questionário de Metas de Realização, reunindo evidências de sua validade fatorial e consistência interna. Antes de considerá-lo, entretanto, parece adequado ter em conta o marco teórico em que se fundamentou.

\section{Motivação e Metas de Realização}

As pessoas diferem em seu nível de atividade, podendo ser, por exemplo, ativas ou passivas, indolentes ou construtivas, sendo que as explicações para tais diferenças não se limitam a fatores biológicos ou disposicionais. Os fatores motivacionais têm também um papel preponderante fundamentando as escolhas, a orientação a objetivos, o esforço e a perseverança na ação (Guimarães \& Bzuneck, 2002; Pintrich \& Schunk, 1996). A motivação é também um elemento central no âmbito das teorias acerca das metas de realização (Murphy \& Alexander, 2000; Pintrich, 2000).

As metas de realização referem-se a proposições ou razões de indivíduos que possuem uma tarefa a realizar. Esse construto parece ser operacionalizado melhor em termos de atividades de aprendizagem acadêmica, embora possa ser aplicado a outros contextos de realização (por exemplo, no âmbito do trabalho, dos negócios; Pintrich \& Schunk, 1996). Na escola, a motivação tem sido considerada um determinante crítico do nível e da qualidade de aprendizagem e desempenho, constituindo-se, portanto, em um propósito valioso a ser buscado na área educacional (Guimarães \& Bzuneck, 2002). Segundo Guimarães e Boruchovitch (2004), um estudante motivado mostrase ativamente envolvido no processo de aprendizagem, engajando-se e persistindo em tarefas desafiadoras, despendendo esforços, usando estratégias adequadas, buscando desenvolver habilidades de compreensão e domínio. Além disso, apresenta entusiasmo na execução de tarefas e orgulho acerca dos seus resultados, podendo superar previsões baseadas apenas em suas habilidades ou seus conhecimentos prévios.

A compreensão do desempenho escolar e das estratégias de estudo adotadas por alunos constitui um aparato de grande importância no contexto escolar. Neste sentido, as pesquisas acerca das metas de realização devem ser consideradas pertinentes, principalmente no que se refere aos estudantes do ensino médio, prestes a submeteremse ao vestibular e a decidir por uma profissão. No caso das pesquisas de Elliot e seus colaboradores (e.g., Elliot \& Church, 1997; Elliot \& McGregor, 1999; Elliot \& cols., 1999), tornam-se relevantes não apenas por razões teóricas, mas também do ponto de vista de práticas pedagógicas. Ao entender que os estudantes não são somente motivados em termos de quantidade, mas que, de fato, existem importantes diferenças qualitativas em como são motivados, reconhece-se que essas diferenças têm uma influência evidente na aprendizagem e realização profissional. A teoria e a medida elaboradas por esses autores são descritas a seguir.

\section{O Modelo 2 x 2 de Elliot e McGregor: Teoria e Medida}

Existem diferentes modelos acerca das metas de reali-

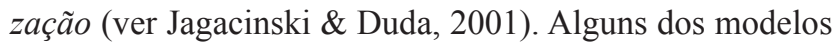
teóricos assumem que as metas são mais determinadas em função de fatores contextuais (por exemplo, estrutura de salas de aula), sendo assim mais maleáveis (Puca \& Schmalt, 1999). Outros, entretanto, tratam das metas de realização como sendo influenciadas fortemente por características individuais e pessoais, definindo-se como mais estáveis (Elliot, 1999; Elliot \& Church, 1997; Elliot \& Harackiewicz, 1996; Elliot \& Trash, 2002; Pintrich, 2000). Esta última perspectiva é adotada aqui, embasando o modelo de Elliot.

Apesar da diversidade de modelos teóricos, previamente assinalada, parece haver acordo acerca de duas dimensões principais, conceituadas a partir de como se define e avalia a competência. Especificamente, de acordo com Elliot e McGregor (2001), a competência é definida em termos do referente que é usado para julgar o desempenho, resultando em duas orientações principais - absoluta/intrapessoal (o indivíduo busca conhecer a tarefa em si ou melhorar seu próprio conhecimento a respeito) e normativa (o indivíduo se empenha em fazer melhor que os demais) -, e é avaliada em termos de uma possibilidade positiva e desejável (sucesso) ou negativa e indesejável (fracasso). De acordo com essa concepção, pensaram-se as dimensões de aprendizagem e execução (definição), cruzando-as com aquelas de aproximação e evitação (avaliação), sendo propostas as seguintes dimensões (Elliot \& McGregor, 2001):

- Meta de aprendizagem/aproximação. Neste tipo de meta (mastery - approach goal), a competência é definida em termos absolutos/intrapessoais e avaliada positivamente. $\mathrm{O}$ foco está na tarefa de maestria, aprendizagem e conhecimento. $\mathrm{O}$ estudante procura se orientar em razão do seu melhoramento e progresso acadêmico, buscando um entendimento profundo da tarefa;

- Meta de aprendizagem/evitação. Este tipo de meta (mastery - avoidance goal) define a competência em termos absolutos/intrapessoais, sendo avaliada negativamente. $\mathrm{O}$ foco está em não obter um entendimento ruim ou deficiente, evitar a não aprendizagem ou a não maestria da tarefa. $\mathrm{O}$ estudante se esforça para não errar, não fazer incorretamente a tarefa.

- Meta de execução/aproximação. Esta meta (performance - approach goal) possibilita definir a competência em termos normativos, sendo avaliada positivamente. $\mathrm{O}$ foco 
está em ser superior, melhor que os outros, ser o mais esperto, melhor na tarefa em comparação aos demais. O estudante guia-se pela obtenção da melhor ou mais alta pontuação, procurando estar no topo ou ter o melhor desempenho da sua sala de aula.

- Meta de execução/evitação. Nesta meta (performance - avoidance goal), a competência é definida em termos normativos, sendo avaliada negativamente. $\mathrm{O}$ foco está em evitar a inferioridade, não ser visto como estúpido em comparação aos outros. $\mathrm{O}$ estudante tem o propósito último de não receber as piores notas, não pretendendo ser considerado como aquele que tem o desempenho mais fraco da sua turma.

Inicialmente, Elliot e seus colaboradores se dedicaram a construir uma medida que se aproximou desse esquema teórico quanto às metas de realização (Elliot \& Church, 1997; Elliot \& cols., 1999). Contudo, consideraram um modelo tricotômico, em que as metas de aprendizagem compreendiam um único fator, sem levar em conta a dimensão avaliativa (aproximação vs. evitação; ver Elliot, 1999). Nesse sentido, apesar de os fatores desse questionário apresentarem parâmetros psicométricos adequados (por exemplo, Alfas de Cronbach superiores a 0,75), a medida correspondente não contemplava a estrutura teórica mais recente (Elliot \& McGregor, 2001).

Portanto, a partir do seu modelo teórico, Elliot e McGregor (2001) propuseram um instrumento específico para medir as quatro metas de realização descritas previamente, denominado de Achievement Goals Questionnaire. Este instrumento foi inicialmente construído e validado nos Estados Unidos, considerando amostras de estudantes universitários, e pareceu ser uma medida adequada, apresentando, na oportunidade, parâmetros satisfatórios. Por exemplo, todos seus 12 itens apresentaram saturações acima de 0,70 (Componentes Principais), independente do tipo de rotação, e consistência interna (Alfa de Cronbach) variando de 0,83 (meta de execução-evitação) a 0,92 (meta de execução-aproximação). Uma análise fatorial confirmatória $(M L)$ indicou ajuste adequado desse modelo com quatro fatores: $\chi^{2}(48, N=148)=60,49$; $p=0,11 ; C F I=0,99 ; T L I=0,99$ e $R M S E A=0,042$. Finney, Pieper e Barron (2004) testaram essa medida em outra amostra de estudantes universitários estadunidenses $(N=2.111)$, relatando resultados parecidos aos da versão original (por exemplo, $C F I=0,95$ e $R M S E A=0,066)$.

Esse instrumento não é, não obstante, o único disponível na literatura para medir metas de realização. Jagacinski e Duda (2001) apresentaram e compararam três de tais medidas, todas apresentando índices aceitáveis de consistência interna (Alfa de Cronbach; amplitude de 0,80 a 0,92), porém centrando-se na dimensão definição e avaliando principalmente duas orientações principais: metas de execução (habilidade e ego) e metas de maestria (tarefa e aprendizagem). Essas medidas não têm tido em conta a dimensão avaliativa, correspondendo às orientações de aproximação e evitação, como contempladas por Elliot e McGregor (2001).

Em resumo, pretende-se com o presente estudo conhecer evidências de validade fatorial e consistência interna do Questionário de Metas de Realização para o contexto brasileiro. Além disso, procura-se caracterizar as pontuações dos participantes deste estudo e correlacioná-las com indicadores de desempenho escolar.

\section{Método}

\section{Participantes}

Participaram da pesquisa 307 estudantes do ensino médio de duas escolas públicas (68\%) e uma particular (32\%) da cidade de João Pessoa. Esses tinham idades de 13 a 52 anos $(M=17,6 ; D P=3,94 ; 90 \%$ tinham até 20 anos de idade), distribuídos entre $1^{\circ}$ ano $(41 \%), 2^{\circ}$ ano $(26 \%)$ e $3^{\circ}$ ano $(33 \%)$, sendo a maioria do sexo feminino $(61,2 \%)$. Em comparação com os demais colegas de sala, tais indivíduos majoritariamente se percebiam como "bons estudantes" (34\%), com pontuação média nessa avaliação $(M=5,1$; $D P=1,07)$ acima da mediana teórica da escala de resposta (4; amplitude de 1=Péssimo a 7=Ótimo). Esses indivíduos relataram que no último ano escolar obtiveram médias 7,9 e 7,5 em Português e Matemática, respectivamente. A maioria disse dedicar em média 3 horas por dia ao estudo (62,7\%), excetuando as horas de sala de aula. Essa foi uma amostra de conveniência, não-probabilística; foram incluídos aqueles jovens que, contatados em sala de aula, aceitaram participar do estudo.

\section{Instrumentos}

Os participantes receberam uma folha impressa, com as seguintes partes:

- Questionário de Metas de Realização-QMR. Elaborado originalmente em língua inglesa, especificamente no contexto estadunidense, por Elliot e McGregor (2001), compõe-se de 12 itens. Esses são eqüitativamente distribuídos em quatro metas de realização que foram teoricamente derivadas: aprendizagem-aproximação (por exemplo, Desejo dominar completamente o assunto apresentado na sala de aula), aprendizagem-evitação [por exemplo, Às vezes fico receoso (com medo) de não poder compreender o conteúdo das aulas como eu gostaria], execução-aproximação (por exemplo, Minha meta nesta sala é conseguir notas melhores do que a maioria dos estudantes) e execução-evitação (por exemplo, Meu objetivo é evitar ter um desempenho ruim nas aulas). Os itens são respondidos em escala de 7 pontos, com os seguintes extremos: 1 = Não me descreve nada e 7 = Descreve-me totalmente

A tradução da versão em inglês do $Q R M$ para o português foi realizada por meio do método back translation. Com a versão preliminar na língua portuguesa, prosseguiu-se a sua validação semântica, tendo sido considerado um grupo de 20 participantes do $1^{\circ}$ ano do ensino médio. Esse procedimento visou dirimir quaisquer dificuldades de compreensão dos participantes em relação ao questionário como um todo, bem como acerca das instruções e do formato de resposta. Nenhum item precisou ser modificado, sendo possível respondê-los na escala de resposta proposta.

- Indicadores de Desempenho Escolar. Incluíram-se perguntas relacionadas com as notas em Português e Matemática que os participantes obtiveram no ano escolar anterior à coleta de dados, bem como acerca do tempo que dedicam aos estudos extra-sala de aula, se desejam fazer algum curso universitário e sua autopercepção, em comparação com os seus pares, do quão são bons estudantes. 
- Caracterização da amostra. Foram realizadas perguntas de caráter demográfico (por exemplo, idade, sexo) para descrição da amostra.

\section{Procedimento}

Inicialmente, contataram-se os diretores das instituições de ensino de João Pessoa. A esses foram explicitados os objetivos da pesquisa e, uma vez obtida sua permissão, combinou-se o horário melhor para a aplicação dos questionários. Buscando atender os aspectos éticos que norteiam o procedimento em pesquisa com seres humanos, os diretores das instituições de ensino assinaram um termo de consentimento livre e esclarecido autorizando a participação dos alunos. A coleta de dados se deu em horário de aula, no próprio ambiente escolar. Embora a coleta tenha sido feita em contexto coletivo, solicitou-se que os participantes respondessem individualmente. Foram recrutados quatro aplicadores, igualmente distribuídos quanto ao sexo. Todos os instrumentos foram de auto-aplicação, exigindo o mínimo de interferência por parte do seu aplicador. Estabeleceu-se que apenas em caso de falta de compreensão sobre como responder ou alguma outra questão de forma, os aplicadores poderiam auxiliar os respondentes. A todos foi informado que se tratava de um estudo cuja participação seria voluntária, sendo assegurado o sigilo das respostas, tratadas coletiva e estatisticamente. Os participantes levaram cerca de 20 minutos para responder ao questionário.

\section{Análise de dados}

O SPSS (versão 13) foi utilizado para tabular e analisar os dados. Além de estatísticas descritivas (medidas de tendência central, dispersão, distribuição de freqüência), calcularam-se o índice KMO e o Teste de Esfericidade de Bartlett com o propósito de conhecer se a matriz de correlação entre as 12 metas era fatorializável. Fez-se ainda uma análise de Componentes Principais, sem fixar o número de fatores, e uma análise paralela. Nesse caso, realizaram-se 1.000 simulações, considerando os parâmetros do banco de dados original (12 variáveis, 307 participantes). Com o fim de comparar as pontuações nos componentes de metas de realização, efetuou-se uma MANOVA para medidas emparelhadas (repetidas).

Por meio do AMOS (versão 4) foram realizadas múltiplas análises fatoriais confirmatórias, testando o modelo teórico proposto e comparando-o com modelos alternativos. Em todos os casos, considerou-se o método da Máxima Verossimilhança $(M L)$, tratando diretamente com a matriz de covariância. Em relação aos indicadores de adequação do modelo (índices de ajuste) aos dados empíricos, os seguintes são comumente considerados (Byrne, 2001; Kelloway, 1998): o adjusted goodness-of-fit index (AGFI), o comparative fit index $(C F I)$ e a root-mean-square error of approximation (RMSEA), com seu intervalo de confiança de $90 \%$ (IC). Modelos com AGFI e CFI de 0,90 ou mais indicam ajustes aceitáveis; no caso da RMSEA, um valor próximo a 0,06 ou menos revela um ajuste aceitável, admitindo-se valores de até 0,10 (Browne \& Cudeck, 1993; Hu \& Bentler, 1999). O expected cross-validation index (ECVI) e o consistent Akaike information criterion
(CAIC) foram também calculados, servindo para comparar modelos alternativos; quanto menor forem os respectivos valores, mais ajustado será o modelo (Fabrigar, Wegener, MacCallum, \& Strahan, 1999; Garson, 2003). Finalmente, teve-se em conta a diferença dos qui-quadrados e seus respectivos graus de liberdade $\left[\Delta \chi^{2}(\mathrm{gl})\right]$ para decidir acerca do melhor ajuste do modelo. No caso, havendo diferença significativa, o melhor modelo corresponde ao que apresenta menor qui-quadrado (Garson, 2003).

\section{Resultados}

\section{Análise fatorial exploratória - PC}

Com o fim de decidir acerca da adequação de se realizar uma análise fatorial com a matriz de correlação correspondente aos 12 itens do $Q M R$, tomaram-se como referência o $K M O(0,72)$ e o Teste de Esfericidade de Bartlett $\left[\chi^{2}(66)=668,352 ; p<0,001\right]$, que apóiam essa análise. No caso do primeiro, consideram-se as correlações parciais entre os itens, devendo ser aceitos índices iguais ou superiores a 0,60 . Por outro lado, o segundo comprova a hipótese de que a matriz de covariância é uma matriz de identidade, com valores significativos $(p<0,05)$ indicando que esta deve ser rejeitada e, por conseguinte, endossando a pertinência de se proceder adiante (Tabachnick \& Fidell, 1996).

Na oportunidade, procurou-se ainda checar quantos fatores/componentes poderiam ser adequadamente identificados na matriz de intercorrelações entre os itens, tendo-se em conta três critérios principais: (1) Kaiser (valor próprio maior do que 1); (2) Cattell (distribuição gráfica dos valores próprios, scree plot); e (3) análise paralela. Embora os dois primeiros venham sendo amplamente usados (Cattell \& Krug, 1986), reconhecidamente o último critério é mais confiável (Hayton, Allen \& Scarpello, 2004). Em todo caso, a interpretabilidade da solução fatorial foi igualmente decisiva para indicar o número de fatores a extrair. Na Figura 1 são resumidos os resultados dos critérios estatísticos.

A análise de Componentes Principais indicou até quatro componentes com valores próprios superiores a 1 (Critério de Kaiser), explicando conjuntamente 59,2\% da variância total. De acordo com a linha tracejada, nitidamente três ou quatro componentes podem ser retirados (Critério de Cattell). Conforme a análise paralela, a partir do quarto componente o valor próprio observado $(1,03)$ é inferior àqueles simulados, no tocante às médias dos valores próprios $(1,12)$ ou ao percentil 95\% $(1,16)$. Isso sugere extrair três componentes. Contudo, em razão do marco teórico considerado e da interpretabilidade da estrutura resultante, decidiu-se assumir os quatro componentes, descritos na Tabela 1.

Segundo se observa nessa tabela, no geral, os quatro componentes emergem em consonância com a teoria que os fundamenta. Procura-se a seguir descrever e interpretar cada um deles.

\section{Componente I}

Este primeiro componente apresentou valor próprio de 2,12 , explicando $17,7 \%$ da variância total. Todos os itens pertencentes à dimensão aprendizagem-evitação 


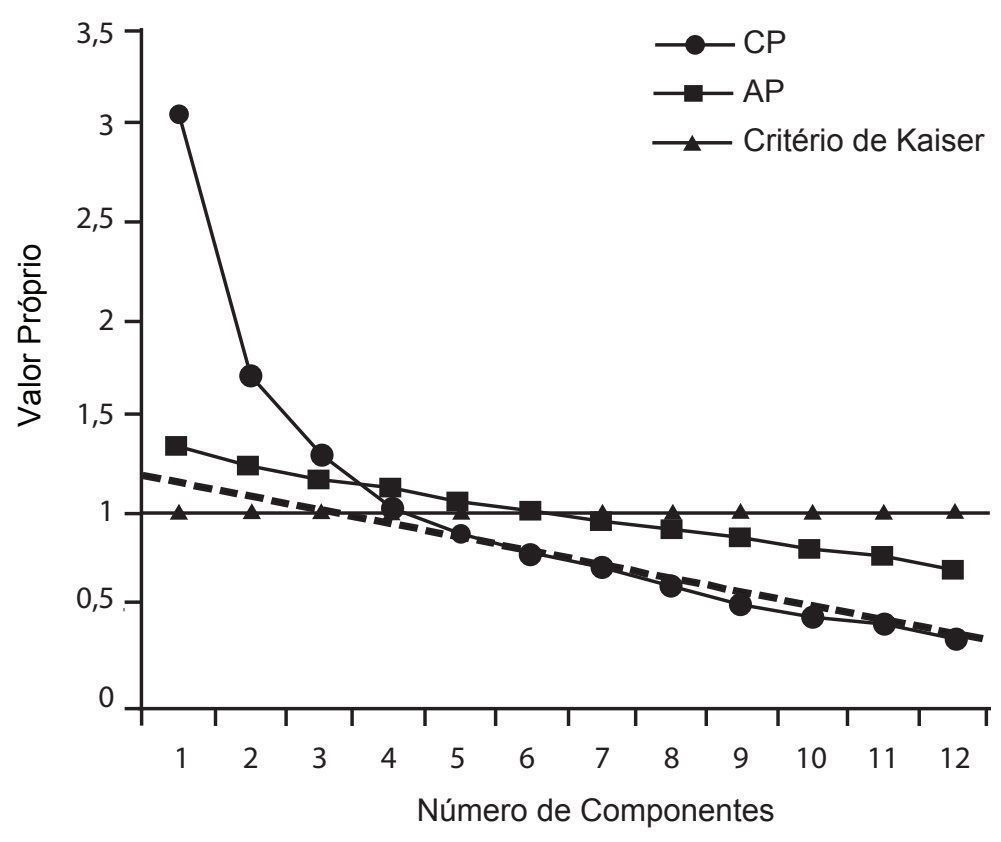

Figura 1. Distribuição gráfica dos valores próprios e o número de componentes a extrair $(\mathrm{CP}=$ Componentes Principais; $\mathrm{AP}=\mathrm{Análise} \mathrm{Paralela})$.

Tabela 1. Estrutura fatorial do Questionário de Metas de Realização.

\begin{tabular}{|c|c|c|c|c|c|}
\hline \multirow[b]{2}{*}{ Item } & \multirow[b]{2}{*}{ Conteúdo } & \multicolumn{4}{|c|}{ Componentes } \\
\hline & & I & II & III & IV \\
\hline 02. & $\begin{array}{l}\text { Às vezes fico receoso (com medo) de não poder compreender o conteúdo das aulas } \\
\text { como eu gostaria }\end{array}$ & $0,76^{*}$ & 0,03 & $-0,01$ & 0,04 \\
\hline 07. & $\begin{array}{l}\text { Preocupo-me freqüentemente em não poder aprender tudo o que há para aprender na } \\
\text { sala de aula. }\end{array}$ & $0,76^{*}$ & 0,24 & 0,18 & $-0,18$ \\
\hline 12. & Preocupa-me que eu não consiga aprender tudo que poderia na sala de aula. & $0,75^{*}$ & 0,01 & 0,16 & 0,15 \\
\hline 01. & É importante, para mim, fazer melhor do que os demais estudantes desta sala de aula. & 0,01 & $0,81 *$ & 0,10 & 0,08 \\
\hline 10. & $\begin{array}{l}\text { Minha meta nesta sala de aula é conseguir notas melhores do que a maioria dos } \\
\text { estudantes. }\end{array}$ & 0,13 & $0,80^{*}$ & 0,10 & $-0,04$ \\
\hline 05. & $\begin{array}{l}\text { É importante, para mim, ter um bom desempenho quando comparado com os demais } \\
\text { colegas de sala de aula. }\end{array}$ & 0,09 & $0,76^{*}$ & 0,16 & 0,09 \\
\hline 03. & Desejo dominar completamente o assunto apresentado na sala de aula. & 0,04 & 0,07 & $0,75^{*}$ & $-0,03$ \\
\hline 09. & Quero aprender tanto quanto possível nas aulas. & 0,06 & 0,04 & $0,69 *$ & 0,18 \\
\hline 06. & É importante, para mim, compreender o máximo possível o conteúdo das disciplinas. & 0,10 & 0,33 & $0,68 *$ & $-0,05$ \\
\hline 08. & Meu objetivo é evitar ter um desempenho ruim nas aulas. & 0,05 & $-0,03$ & 0,22 & $0,86^{*}$ \\
\hline 04. & O que me motiva em sala de aula é meu medo de ter um desempenho ruim. & 0,34 & 0,23 & $-0,24$ & $0,50^{*}$ \\
\hline \multirow[t]{5}{*}{11.} & Quero apenas evitar me sair mal (ter um desempenho fraco) na sala de aula. & 0,05 & 0,02 & $-0,02$ & $0,26^{*}$ \\
\hline & Número de Itens & 3 & 3 & 3 & 3 \\
\hline & Valor Próprio & 2,12 & 2,09 & 1,71 & 1,16 \\
\hline & Alfa de Cronbach & 0,70 & 0,74 & 0,60 & 0,32 \\
\hline & \% Variância Total & 17,7 & 17,5 & 14,3 & 9,7 \\
\hline
\end{tabular}


apareceram neste componente, com saturações variando de 0,75 (Preocupa-me que eu não consiga aprender tudo que poderia na sala de aula) a 0,76 (Preocupo-me freqüentemente em não poder aprender tudo o que há para aprender na sala de aula), o que não deixa dúvidas quanto a sua interpretação. Um item da dimensão execução-evitação também saturou nesse componente (0,34; O que me motiva em sala de aula é meu medo de ter um desempenho ruim), mas não foi levado em conta para o cálculo da pontuação deste componente, cuja consistência interna (Alfa de Cronbach) foi de 0,70 .

\section{Componente II}

Este componente mostrou um valor próprio igual a 2,09, explicando $17,5 \%$ da variância total. Os três itens com maiores saturações representam a dimensão execução-aproximação, variando de 0,76 (É importante, para mim, ter um bom desempenho quando comparado com os demais colegas de sala de aula) a 0,81 (É importante, para mim, fazer melhor do que os demais estudantes desta sala de aula). O item $E$ importante, para mim, compreender o máximo possível o conteúdo das disciplinas apresentou saturação de 0,33 neste componente, embora represente mais adequadamente aquele de aprendizagem-aproximação. O Alfa de Cronbach deste segundo componente foi 0,74 .

\section{Componente III}

Os três itens reunidos por este componente pertencem exclusivamente à dimensão aprendizagem-aproximação; seu valor próprio foi 1,71, correspondendo à explicação de 14,3\% da variância total. A menor e maior saturações corresponderam, respectivamente, aos seguintes itens: $E$ importante, para mim, compreender o máximo possível o conteúdo das disciplinas $(0,68)$ e Desejo dominar completamente o assunto apresentado na sala de aula $(0,75)$. Este apresentou um Alfa de Cronbach de 0,60.

\section{Componente IV}

Dos três itens que deveriam representar a dimensão execução-evitação, apenas um apresentou saturação inferior a 0,50: Quero apenas evitar me sair mal (ter um desempenho fraco) na sala de aula [0,26; estatisticamente diferente de zero, $t(208)=4,72 ; \mathrm{p}<0,001]$. Os dois restantes foram: O que me motiva em sala de aula é meu medo de ter um desempenho ruim $(0,50)$ e Meu objetivo é evitar ter um desempenho ruim nas aulas $(0,86)$. Portanto, é possível interpretar este componente como consistente com a dimensão referida. Este apresentou valor próprio de 1,19, explicando 9,7\% da variância total, com Alfa de Cronbach de 0,32.

Em resumo, com exceção de um item no quarto componente, todos os demais apresentaram saturações iguais ou superiores a 0,50 na dimensão em que eram teoricamente esperados. A consistência interna desse componente foi também a mais discrepante. Contudo, no conjunto, os resultados parecem bem consistentes, sugerindo evidências favoráveis ao menos de validade fatorial do Questionário de Metas de
Realização. A propósito desse ponto, caberia especular se o modelo observado é mesmo adequado, comparando-o com modelos alternativos. Nesse sentido, realizaram-se novas análises, como descritas a seguir.

\section{Comparação de modelos alternativos da estrutura das metas de realização}

Com o fim de proceder à modelagem por equações estruturais, decidiu-se inicialmente eliminar do banco de itens 10 casos que apresentaram ao menos um item sem resposta $(3,2 \%$; missing). Feito isso, procedeu-se a testar cinco modelos alternativos: Modelo 1: assumiu-se uma estrutura unifatorial, em que todos os itens saturam em um mesmo fator; Modelo 2: os itens saturando em dois fatores de primeira ordem, correspondendo aos tipos de definições (aprendizagem e execução); Modelo 3: os itens saturando em dois fatores de primeira ordem, correspondendo aos tipos de avaliações (aproximação e evitação); Modelo 4: foram admitidos quatro fatores de primeira ordem, correspondente aos tipos de metas motivacionais, e dois de segunda ordem, que retratam os tipos de definições dessas metas (aprendizagem e execução); Modelo 5: também foram admitidos os mesmos quatro fatores de primeira ordem do modelo anterior, porém com os dois fatores de segunda ordem correspondendo aos tipos de avaliações (aproximação e evitação); e, finalmente, Modelo 6: admitiram-se apenas os quatro fatores de primeira ordem, conforme sugere o modelo teórico testado. Os resultados a respeito são sumarizados na Tabela 2 .

De acordo com essa tabela, percebe-se que o Modelo 1 (unifatorial) é o menos adequado de todos, sendo perfeitamente rejeitado. Procurou-se tomá-lo como referência, contrastando o seu qui-quadrado com o dos modelos seguintes $\left(\Delta \chi^{2}\right)$. O Modelo 2 procurou agrupar os itens em dois fatores de primeira ordem, segundo os tipos de definições (aprendizagem e execução), com indicadores de ajustes mais satisfatórios que o modelo anterior, contudo longe do que seriam valores desejáveis. O Modelo 3, que reuniu os itens em dois fatores, correspondendo aos tipos de avaliações (aproximação e afastamento), representa uma melhora em comparação com o anterior, mas ainda não cumpre o que tem sido aceito na literatura. O Modelo 4, que considerou como fatores de segunda ordem os tipos de definições das metas é apenas meritório, com valores de CFI e RSMEA considerados marginais. Esse modelo é estatisticamente mais satisfatório que o anterior, contudo seu $\chi^{2}$ é superior aos dois últimos modelos. O Modelo 5 considerou os quatro fatores originais de primeira ordem, procurando agrupá-los em dois de segunda ordem, segundo os tipos de avaliações (aproximação e afastamento). Todos os seus indicadores de ajuste estão dentro do que é recomendado, podendo ser defensável como um modelo adequado aos dados. Resultado semelhante é observado para o Modelo 6, que foi o teoricamente proposto. A comparação desses dois modelos não revelou qualquer diferença significativa, $\Delta \chi^{2}(1)=2,36 ; p>0,05$. Portanto, este último modelo é aqui admitido, sendo teoricamente consistente e mais parcimonioso que o anterior, corroborando a existência de apenas quatro fatores de primeira ordem. 
Tabela 2. Comparação de diferentes modelos fatoriais do Questionário de Metas de Realização.

\begin{tabular}{cccccccc}
\hline Modelo & $\boldsymbol{\chi}^{\mathbf{2}}(\mathbf{g l})$ & AGFI & CFI & RSMEA (CI90\%) & ECVI & CAIC & $\Delta \boldsymbol{\chi}^{\mathbf{2}}(\mathbf{g l})$ \\
\hline 1 & $311,09(54)$ & 0,75 & 0,58 & $0,13(0,11 ; 0,14)$ & 1,21 & 471,73 & - \\
2 & $233,03(53)$ & 0,81 & 0,71 & $0,11(0,09 ; 0,12)$ & 0,96 & 400,38 & $78,06(1)^{*}$ \\
3 & $160,96(53)$ & 0,87 & 0,82 & $0,08(0,07 ; 0,10)$ & 0,71 & 328,31 & $150,13(1)^{*}$ \\
4 & $117,62(49)$ & 0,91 & 0,89 & $0,07(0,05 ; 0,09)$ & 0,59 & 311,73 & $193,47(5)^{*}$ \\
5 & $99,74(49)$ & 0,92 & 0,92 & $0,06(0,04 ; 0,08)$ & 0,53 & 293,86 & $211,35(5)^{*}$ \\
6 & $97,48(48)$ & 0,92 & 0,92 & $0,06(0,04 ; 0,08)$ & 0,53 & 298,29 & $213,61(6)^{*}$ \\
\hline
\end{tabular}

Notas. $\chi^{2}=$ qui-quadrado, $\mathrm{gl}=$ graus de liberdade, $\mathrm{GFI}=$ goodness-of-fit index, $\mathrm{CFI}=$ comparative fit index, $\mathrm{RMSEA}=$ root-mean-square error of approximation, $\mathrm{CI} 90 \%=$ intervalo de confiança de $90 \%, \mathrm{ECVI}=$ expected cross-validation index, CAIC $=$ consistent Akaike information criterion, $\Delta \chi^{2}=$ diferença entre os valores do qui-quadrado. ${ }^{*} \mathrm{p}<0,001$.

Tabela 3. Estatísticas descritivas e intercorrelações entre os componentes de metas.

\begin{tabular}{|c|c|c|c|c|c|}
\hline Componentes & M & DP & & & \\
\hline 1. Aprendizagem-evitação & 14,8 & 4,64 & & & \\
\hline 2. Execução-aproximação & 12,9 & 5,15 & $0,22 * *$ & & \\
\hline 3. Aprendizagem-aproximação & 18,4 & 2,97 & $0,21 * *$ & $0,32 * *$ & \\
\hline \multirow[t]{2}{*}{ 4. Execução-evitação } & 14,7 & 3,92 & $0,40 * *$ & $0,19 *$ & 0,09 \\
\hline & & & 1 & 2 & 3 \\
\hline
\end{tabular}

Notas. $* \mathrm{p}<0,01, * * \mathrm{p}<0,001$ (bi-caudal; eliminação por pares de casos sem resposta, missing).

Tabela 4. Correlações entre os componentes de metas e indicadores de interesse e/ou desempenho escolar.

\begin{tabular}{lcccc}
\hline \multirow{2}{*}{ Variáveis demográficas } & \multicolumn{4}{c}{ Componentes de metas } \\
\cline { 2 - 5 } & I & II & III & IV \\
\hline Curso universitário & $-0,18^{* *}$ & 0,07 & $-0,17^{* *}$ & 0,02 \\
Bom estudante & $-0,10$ & $0,25^{* * *}$ & $0,22^{* * *}$ & 0,01 \\
Horas de estudo extra sala de aula & $-0,02$ & $0,14^{*}$ & 0,06 & 0,06 \\
Notas português & $-0,07$ & 0,02 & $0,16^{* *}$ & $-0,02$ \\
Notas matemática & $-0,03$ & 0,10 & $0,13^{*}$ & $-0,02$ \\
\hline
\end{tabular}

Notas. I = Aprendizagem-evitação; II = Execução-aproximação; III = Aprendizagem-aproximação; IV = Execução-evitação. * $p<0,05$, ** $p<0,01, * * * p<0,001$ (teste bi-caudal; eliminação por pares de casos sem resposta, missing).

\section{Pontuações dos participantes e correlatos escolares das metas de realização}

Admitindo-se os quatro componentes, criaram-se pontuações totais (soma dos três itens que representam cada um). Posteriormente, procurou-se conhecer como esses componentes se correlacionavam entre si e em quais os participantes obtinham maiores pontuações. Na Tabela 3 oferece-se um resumo a respeito.

Conforme se observa nessa tabela, unicamente o componente execução-evitação não se correlacionou significativamente com um dos demais componentes $(r=0,09$; $p>0,05$; aprendizagem-aproximação). Todos os demais o fizeram diretamente $(p<0,01)$, com correlações variando de 0,19 (execução-aproximação e execução-evitação) a 0,40 (aprendizagem-evitação e execução-evitação). Uma
MANOVA para medidas emparelhadas indica que existe diferença nas pontuações dos participantes nesses quatro componentes [Lambda de Wilks $=0,40 ; F(3,294)=147,37$; $p<0,001]$. Especificamente, sua pontuação é maior na meta de aprendizagem-aproximação $(M=18,4)$, estatisticamente superior (teste post hoc de Bonferroni) àquelas em aprendizagem-evitação $(M=14,8)$ e execução-evitação $(M=14,7)$ - essas duas não se diferenciaram entre si-e, finalmente, em execução-aproximação $(M=12,9)$. Esta foi inferior a todas as demais pontuações.

Finalmente, correlacionaram-se as pontuações de metas de realização com cinco indicadores de interesse e/ou desempenho escolar. Os resultados correspondentes podem ser observados na Tabela 4.

De acordo com essa tabela, as pontuações na meta de aprendizagem-evitação se correlacionaram inversamente com 
o desejo de fazer um curso universitário $(r=-0,18 ; p<0,01)$; as de execução-aproximação o fizeram diretamente com o quanto o participante se considera um bom estudante $(r=0,25 ; p<0,001) \mathrm{e}$ com as horas que dedica ao estudo fora da sala de aula $(r=0,14$; $p<0,05)$; aquelas de aprendizagem-aproximação apresentaram correlação direta com três dos indicadores: o quanto se considera um bom estudante $(r=0,22 ; p<0,001)$, as notas obtidas (informadas) nas disciplinas de Português $(r=0,16 ; p<0,01)$ e Matemática $(r=0,13 ; p<0,05)$, tendo correlação inversa com o desejo de fazer curso superior $(r=-0,17 ; p<0,01)$. Nenhuma correlação significativa foi observada da meta de execução-evitação com esse conjunto de indicadores escolares.

\section{Discussão}

Estima-se que o propósito principal desta pesquisa, que consistiu em conhecer a validade fatorial e a consistência interna do Questionário de Metas de Realização (Elliot \& McGregor, 2001), tenha sido satisfatoriamente alcançado. Entretanto, cabe reconhecer potenciais limitações deste estudo, especialmente no que diz respeito aos participantes. Tratou-se de uma amostra não probabilística e específica (estudantes do ensino médio), não sendo numericamente representativa da população brasileira ou mesmo pessoense. Aponta-se, ainda, que o que se sabe previamente sobre essa medida tomou como referência estudantes universitários, o que pode explicar eventuais discrepâncias entre os resultados obtidos. Não obstante, essas restrições precisam ser ponderadas, considerando-se que o propósito do presente estudo não foi generalizar os resultados, mas conhecer evidências de sua validade fatorial e consistência interna no contexto em que foi aplicado. Discutem-se a seguir esses parâmetros.

\section{Estrutura fatorial e confiabilidade}

Teoricamente, o Questionário de Metas de Realização reúne quatro componentes ou fatores principais, tendo sido comprovada essa estrutura com diferentes amostras estadunidenses (Elliot \& McGregor, 2001; Finney \& cols., 2004). Critérios convencionais, a exemplo daqueles de Kaiser e Cattell (Cattell \& Krug, 1986), revelaram ser pertinente extrair esse número de componentes; um critério mais robusto como a análise paralela (Hayton \& cols., 2004), contudo, sugeriu três componentes. Porém, o marco teórico e a interpretabilidade do conjunto de componentes favoreceram optar pelos quatro originalmente previstos. Excetuando o quarto componente, correspondendo à execução-evitação, em todos os demais, os itens que teoricamente os definiam figuraram com saturações de 0,50 ou mais, reforçando a validade fatorial da estrutura teórica proposta.

Apesar do antes comentado, caberia uma prova mais substancial a respeito da estrutura da medida de metas de realização, que se obteve a partir de análises fatoriais confirmatórias. Comparando os diversos modelos, pareceu bastante notória a estrutura com quatro fatores de primeira ordem, como prevista; uma estrutura alternativa poderia ser também admitida, compreendendo esses mesmos quatro fatores e dois de segunda ordem (aproximação e evitação). Porém, por ser mais parcimonioso, o modelo anterior foi preterido a esse último. Ambos, entretanto, apresentam indicadores de ajuste que cumprem os valores convencionalmente estabelecidos na literatura (Browne \& Cudeck, 1993; Fabrigar \& cols., 1999; Garson, 2003; Hu \& Bentler, 1999). Esses indicadores foram muito similares aos observados previamente, ainda que em outra cultura e considerando um grupo de participantes com características demográficas diferentes das aqui descritas (Elliot \& McGregor, 2001; Finney \& cols., 2004).

Embora tenha sido evidenciada a qualidade psicométrica de validade desse instrumento, os resultados foram menos promissores no que se refere à sua confiabilidade ou propriamente consistência interna. No caso do estudo em que essa medida foi apresentada, seus fatores tiveram coeficientes acima de 0,80; o que apresentou o menor Alfa de Cronbach foi o correspondente às metas de execução-evitação $(0,83$; Elliot \& McGregor, 2001). Consistentemente, na presente pesquisa esse também foi o fator com menor consistência interna, porém bem abaixo do 0,70 que tem sido convencionalmente recomendado na literatura (Nunnally, 1987/1991; Pasquali, 2003). Cabem aqui duas ponderações: (1) apesar de ter apresentado Alfa de Cronbach acima de 0,80 no estudo de sua elaboração, o fator execução-evitação, como todos os demais, é formado por apenas três itens, sendo o menor número de itens desfavorável para esse índice. Se, por exemplo, esse instrumento contasse com mais sete itens com as mesmas características métricas que os demais, poder-se-ia observar um Alfa de 0,61 (fórmula 7.6, Nunnally, 1987/1991, p. 267); (2) a maioria dos Alfas de Cronbach verificados no presente estudo para essa medida, iguais ou superiores a 0,60 , pode ser considerada adequada quando o propósito último de seu emprego for para fins de pesquisa (Clark \& Watson, 1995; Mueller, 1986). Esses aspectos reforçam pensar que, apesar de não terem sido excepcionais, os índices de confiabilidade são promissores e justificáveis.

\section{Metas de realização e rendimento acadêmico}

Como observado no estudo de Elliot e McGregor (2001), no geral, as metas de realização se correlacionaram positivamente entre si. Isso, como ficou demonstrado previamente, não sugere que representem um mesmo fator ou componente; são, em realidade, quatro dimensões de um mesmo construto motivacional, isto é, metas de realização (Murphy \& Alexander, 2000; Pintrich, 2000). Esse fato não faz com que as metas sejam indistintas, ou seja, que compartilhem um mesmo sentido ou foco. Elas têm especificidades e importâncias variadas em detrimento de algumas pessoas e, provavelmente, contextos sociais. No contexto do ensino médio, seguramente não é surpresa a maior pontuação dos participantes em metas de aprendizagem-aproximação. Estas têm sido apontadas na literatura como cruciais para o bom desempenho escolar (Dupeyrat \& Mariné, 2005; Elliot \& Church, 1997; Elliot \& McGregor, 1999, 2001; Elliot \& cols., 1999; Finney \& cols., 2004).

Coerentemente com o indicado, observou-se que os participantes que pontuaram alto em metas de aprendizagem-aproximação relataram maiores notas em Português e Matemática. Porém, esses resultados não são unânimes. A propósito, Bouffard, Boisvert, Vezeu e Larouche (1995) observaram que o desempenho acadêmico pode estar relacionado tanto com as metas de aprendizagem como com 
aquelas de execução. De acordo com Harackiewicz, Barron, Carter, Lehto e Elliot (1997), seriam as pontuações altas nas metas de execução que estariam relacionadas com as maiores notas no exame final da disciplina focalizada em seu estudo. Contudo, diferentemente destes autores, Zenorini e cols. (2003) apontam que as metas de execução, tanto na sua dimensão aproximação como evitação, correlacionam-se negativamente com notas escolares.

Os resultados sobre a relação das metas de realização e o desempenho escolar carecem mesmo de uma análise sistemática cuidadosa. Enquanto algumas pesquisas assinalam os efeitos prejudiciais da meta de execução (Ames, 1992; Archer, 1994; Dweck \& Elliott, 1983), outros revelam seus efeitos positivos, principalmente quando interagindo com a orientação à meta de aprendizagem. Por exemplo, a pesquisa realizada com estudantes universitários por Bouffard e cols. (1995) mostrou que os alunos com pontuações altas em ambos os tipos de meta (aprendizagem e execução) apresentaram maior uso de estratégias cognitivas e metacognitivas, e um desempenho acadêmico melhor.

Em acordo com a literatura, verificou-se uma correlação direta entre as metas de execução-aproximação e autopercepção dos participantes como bons estudantes e a quantidade de horas estudadas por estes, isto é, aqueles que apresentam tais metas se esforçam mais e têm, conseqüentemente, bom desempenho (McGregor \& Elliot, 2002). Não obstante, o fato de os estudantes se orientarem a partir da comparação social com os demais colegas de sala é algo que merecerá atenção no futuro, uma vez que aqueles estudantes que se pautam por esse tipo de meta se revelam mais ansiosos, o que, seguramente, pode ser um agravante para o fracasso e o desempenho escolar insatisfatório (Gouveia, Gaião e Barbosa, Gouveia \& Barbosa, 2004). Diferentemente, aqueles que se pautam por metas de aprendizagem e apresentam maiores expectativas de competência sentem-se mais confiantes e seguros, podendo ser isso determinante para se saírem melhor nos exames escolares (Dupeyrat \& Mariné, 2005).

\section{Considerações Finais}

Embora tenha sido identificado um problema no quarto componente da análise fatorial exploratória, as análises fatoriais confirmatórias comprovaram a adequação do modelo teórico de Elliot e McGregor (2001). Sugerem-se, porém, novas pesquisas com o intuito de assegurar a consistência interna da subescala execução-evitação. Nesse caso, poderse-ia rever a redação do item 11 ou criar novos itens que representem a dimensão em questão.

Finalmente, seriam igualmente relevantes estudos futuros que visassem conhecer em que medida as metas de realização poderiam estar associadas com outros atributos psicológicos. Por exemplo, poder-se-ia verificar os tipos de metas predominantes em cada série/ano escolar e sua influência no ajustamento escolar, principalmente no que diz respeito à transição do ensino fundamental para o médio. Caberia também conhecer seus correlatos valorativos, isso é, em que medida os valores que guiam a vida dos jovens poderiam se associar com os tipos de metas adotados, ou mesmo se seriam relacionados com os estilos parentais. Portanto, recomenda- se que o Questionário de Metas de Realização seja utilizado em pesquisas futuras, servindo como importante instrumento para avaliar o comportamento de indivíduos em tarefas de realização, como aquelas típicas do contexto escolar.

\section{Referências}

Ames, C. (1992). Classrooms: Goals, structures, and student motivation. Journal of Education Psychology, 84, 261-271.

Archer, J. (1994). Achievement goals as a measure of motivation in university students. Contemporary Educational Psychology, 19, 430-460.

Biblioteca Virtual em Saúde - Psicologia. (2006). Metas de realização. Retirado em 15/09/2006 da BVS - Psicologia, http:// www.bvs-psi.org.br/.

Bouffard, T., Boisvert, J., Vezeau, C. \& Larouche, C. (1995). The impact of goal orientation on self-regulation and performance among college students. British Journal of Educational Psychology, 65, 317-29.

Browne, M. W. \& Cudeck, R. (1993). Alternative ways of assessing model fit. Em K. A. Bollen, \& J. S. Long (Eds.), Testing structural equation models (pp. 136-162). Newbury Park: Sage.

Bueno, J. M. H., Zenorini, R. P. C., Santos, A. A. A., Matumoto, A. Y. \& Buchatsky, J. (2007). Investigação das propriedades psicométricas de uma escala de metas de realização. Estudos de Psicologia, 24, 79-87.

Byrne, B. M. (2001). Structural equation modeling with Amos: Basic concepts, applications, and programming. New York: Springer - Verlag.

Cardoso, L. R. \& Bzuneck, J. A. (2004). Motivação no Ensino Superior: metas de realização e estratégias de aprendizagem. Psicologia Escolar e Educacional, 8, 145-155.

Cattell, R. B. \& Krug, S. E. (1986). The number of factor in the 16PF: A review of the evidence with special emphasis on methodological problems. Educational and Psychological Measurement, 46, 509-522.

Clark, L. A. \& Watson, D. (1995). Constructing validity: Basic issues in objective scale development. Psychological Assessment, 7, 309-319.

Covington, M. V. (2000). Goal theory, motivation, and school achievement: An integrative review. Annual Review of Psychology, 51, 171-200.

Dowson, M. \& McInerney, D. M. (2004). The development and validation of the Goal Orientation and Learning Strategies Survey (GOALS-S). Educational and Psychological Measurement, 64, 290-310.

Dupeyrat, C. \& Mariné, C. (2005). Implicit theories of intelligence, goal orientation, cognitive engagement, and achievement: A test of Dweck's model with returning to school adults. Contemporary Educational Psychology, 30, 43-59.

Dweck, C. S. \& Elliott, E. S. (1983). Achievement motivation. Em E. H. Hetheringtton \& P. H. Mussen (Orgs.), Handbook of child psychology: Socialization, personality and social development (pp. 643-692). New York: John Wiley \& Sons.

Elliot, A. (1999). Approach and avoidance motivation and achievement goals. Educational Psychologist, 34, 169-189.

Elliot, A. \& Church, M. (1997). A hierarchical model of approach and avoidance achievement motivation. Journal of Personality and Social Psychology, 72, 218-232. 
Elliot, A. \& Harackiewicz, J. (1996). Approach and avoidance achievement goals and intrinsic motivation: A mediational analysis. Journal of Personality and Social Psychology, 70, 968-980.

Elliot, A. \& McGregor, H. A. (1999). Test anxiety and the hierarchical model of approach and avoidance achievement motivation. Journal of Personality and Social Psychology, 76, 628-644.

Elliot, A. \& McGregor, H. A. (2001). A 2 x 2 achievement goals framework. Journal of Personality and Social Psychology, 80, 501-519.

Elliot, A., McGregor, H. A. \& Gable, S. (1999). Achievement goals, study strategies, and exam performance: A mediational analysis. Journal of Educational Psychology, 91, 549-563.

Elliot, A. \& Thrash, T. M. (2002). Approach-avoidance motivation in personality: Approach and avoidance temperaments and goals. Journal of Personality and Social Psychology, 82, 804-818.

Fabrigar, L. R., Wegener, D. T., MacCallum, R. C. \& Strahan, E. J. (1999). Evaluating the use of exploratory factor analysis in psychological research. Psychological Methods, 4, 272-299.

Finney, S. J., Pieper, S. L. \& Barron, K. E. (2004). Examining the psychometric properties of the Achievement Goal Questionnaire in a general academic context. Educational and Psychological Measurement, 64, 365-382.

Garson, G. D. (2003). PA 765 Statnotes: An online textbook. Retirado em 17/05/2005 do sítio http://www2.chass.ncsu.edu/ garson/pa765/statnote.htm.

Gouveia, R. S. V., Gaião e Barbosa, A. A., Gouveia, V. V. \& Barbosa, G. A. (2004). Depressão infantil e fracasso vs sucesso escolar: Um estudo correlacional. Psico, 35, 115-124.

Guimarães, S. E. R. \& Boruchovitch, E. (2004). O estilo motivacional do professor e a motivação intrínseca dos estudantes: uma perspectiva da teoria da autodeterminação. Psicologia: Reflexão e Crítica, 17,143-150.

Guimarães, S. É. R. \& Bzuneck, J. A. (2002). Propriedades psicométricas de uma medida de avaliação da motivação intrínseca e extrínseca: um estudo exploratório. Psico-USF, 7, 01-08.

Hayton, J. C., Allen, D. G. \& Scarpello, V. (2004). Factor retention decisions in exploratory factor analysis. Organizational Research Methods, 7, 191-205.

Harackiewicz, J. M., Barron, K. E., Carter, S. M., Lehto, A. T. \& Elliot, A. J. (1997). Predictors and consequences of achievement goals in the college classroom: Maintaining interest and making the grade. Journal of Personality and Social Psychology, 73, 1284-1295.

Hu, L.T. \& Bentler, P. M. (1999). Cutoff criteria for fit indexes in covariance structure analysis: Conventional criteria versus new alternatives. Structural Equation Modeling, 6, 1-55.
Jagacinski, C. M. \& Duda, J. L. (2001). A comparative analysis of contemporary achievement goal orientation measures. Educational and Psychological Measurement, 61, 10131039.

Kelloway, E. K. (1998). Using LISREL for structural equation modeling: A researcher's guide. Thousand Oaks, CA: Sage.

McGregor, H. A. \& Elliot, A. J. (2002). Achievement goals as predictors of achievement-relevant processes prior to task engagement. Journal of Educational Psychology, 94, 381-395.

Mueller, D. J. (1986). Measuring social attitudes: A handbook for researchers and practitioners. New York: Teachers College Press.

Murphy, P. K., \& Alexander, P. A. (2000). A motivated exploration of motivation terminology. Contemporary Educational Psychology, 25, 3-53.

Nunnally, J. C. (1991). Teoría psicométrica. (E. M. González, Trad.). México, DF: Trillas. (Trabalho original publicado em 1987)

Pasquali, L. (2003). Psicometria: teoria dos testes na Psicologia e na Educação. Petrópolis, RJ: Vozes.

Pintrich, P. R. (2000). An achievement goal theory perspective on issues in motivation terminology, theory, and research. Contemporary Educational Psychology, 25, 92-104.

Pintrich, P. R. \& Schunk, D. H. (1996). Motivation in education: Theory, research, and applications. Englewood Cliffs, NJ: Prentice Hall.

Pintrich, P. R., Conley, A. M. \& Kempler, T. M. (2003). Current issues in achievement goal theory and research. International Journal of Educational Research, 39, 319-337.

Puca, R. M. \& Schmalt, H. D. (1999). Task enjoyment: A mediator between achievement motives and performance. Motivation and Emotion, 23, 15 - 27.

Tabachnick, B. G. \& Fidell, L. S. (1996). Using multivariate statistics. Needham Heights, MA: Allyn \& Bacon.

Zenorini, R. P. C., Santos, A. A. A. \& Bueno, J. M. H. (2003). Escala de avaliação das metas de realização: Estudo preliminar de validação. Avaliação Psicológica, 2, 165-173. 